\title{
SURFACE PARAMETER ESTIMATION USING BISTATIC POLARIMETRIC X-BAND MEASUREMENTS
}

\section{K. Ben Khadhra ${ }^{1, *}$, T. Boerner ${ }^{2}$, D. Hounam ${ }^{2}$, and M. Chandra ${ }^{3}$}

${ }^{1}$ Interdisciplinary Centre on Climat Change (IC3), Department of Geography and Environmental Management, Faculty of Environment, University of Waterloo, Canada

${ }^{2}$ German Aerospace Center, DLR, Microwaves and Radar Institute, Germany

${ }^{3}$ Dept. of Microwave Engineering and Photonics, Chemnitz University of Technology, Reichenhainerstr, 70, Chemnitz D-09126, Germany

Abstract - The main purpose of this paper is to separately estimate the important surface parameters (soil moisture and roughness) by using full polarimetric bistatic measurements. The results provide a basis for new satellite application of future bistatic measurement systems such as the TanDEM-X satellite mission. Initially, bistatic X-band measurements, which have been recorded in the Bistatic Measurement Facility (BMF) at the DLR Oberpfaffenhofen, Microwaves and Radar Institute, will be presented. The bistatic measurement sets are composed of soils with different well-known statistical roughness scales and different moistures. The BMF has been calibrated using the Isolated Antenna Calibration Technique (IACT). The validation of the calibration was achieved by measuring the reflectivity of fresh water. In the second part, the assessment of the surface parameters (soil moisture and surface roughness) using the well calibrated data introduced in the former related part, will be detailed. The validation of the specular algorithm by estimating the soil moisture of two surfaces with different roughness scales will be reported. Additionally, a new technique using the coherent term of the Integral Equation Method (IEM) to estimate the soil roughness will be presented, as well as the sensitivity of phase and reflectivity with regard to moisture variation and therefore the penetration depth was evaluated. Current results demonstrate a non-linear relationship

Received 1 February 2012, Accepted 23 February 2012, Scheduled 15 March 2012

* Corresponding author: Kais Ben Khadhra (kbenkhad@uwaterloo.ca). 
between the signal phase and the soil moisture, as expected, confirming the possibility of using DInSAR to measure variations in soil moisture.

\section{INTRODUCTION}

Up till now, the microwave remote sensing (air-or space-borne) has been almost exclusively focused on the monostatic geometry. Therefore, most of the current remote sensing methods are still based on backscatter measurements. Actually, for the bistatic case only theoretical methods have been developed and tested with monostatic data. Very few bistatic measurements, with airborne sensors or in controlled anechoic chambers have been reported. Hence, there still remains a vital need to gain experience with and knowledge of bistatic remote sensing methods. Experimental measurements, indoor or outdoor, play a primordial role in investigating new remote sensing methods and in validating surface and volume scattering models. Another purpose of experimental measurements is for supporting conception studies of new remote sensing systems. Hence, a large number of experimental investigations on the backscattering of electromagnetic fields from rough surfaces have been conducted and reported in the last 50 years. These investigations enabled, on the one hand, the improvement of the theoretical models to more accurately assess the roughness and the humidity (via the dielectric constant) of soil and, on the other hand, to develop of new empirical or semi-empirical models, such as the Oh-model [2], or the Dubois model [3]. However, few controlled experimental measurements have been performed for the forward scattering case or the bistatic case. Thus, the different bistatic theoretical models developed so far have been tested and used for the backscattering analysis, or against numerical simulation $[4,5]$. In addition to this, there is still a considerable lack of data aimed at the investigation of the bistatic active remote sensing and its effectiveness in comparison to its monostatic counterpart.

What has been done in bistatic experimental measurements?

The first experimental bistatic measurement was conducted in 1965 by Cost [6] at Ohio State University. The experiment consisted of a series of outdoor measurements with the transmitter and the receiver mounted on two movable truck mounted booms. The targets were different kinds on natural terrain. Only the scattering coefficient (no phase) was measured for a wide range of incidence and departure angles. In 1967, the first airborne bistatic reflection of land and sea was performed by the Applied Electronics Laboratories, Stanmore, Middlesex [7-9]. One aircraft was transmitting a continuous wave 
$(\mathrm{CW})$ in X-band and a receiver was mounted in a second aircraft. Lowresolution images, general pictures over a wide range were produced as results, and three sub-terrain classifications were distinguished: buildings, trees and open grassland. Recently, two other bistatic indoor experimental measurements of rough surfaces have been carried out.

The first one was achieved by Roger De Roo (Michigan University) [10], where different rough surfaces with constant soil moisture have been measured at X-band and validated with different surface scattering models. In the second experiment [11], which was done at the experimental Microwave Signature Laboratory (EMSL), three different rough surfaces with constant soil moisture were measured at different frequencies and validated against different scattering models.

Thus, to the best of our knowledge we can conclude that:

- There is a lack of surface bistatic measurements with different soil moistures,

- There are no experimental investigations to assess the soil parameters (roughness and moisture) for the bistatic case,

- There are up till now no validated models for bistatic scattering.

Therefore, addressing this need, the purpose of this paper is to establish a basis of a bistatic radar remote sensing system for surface parameter measurements.

\section{BISTATIC MEASUREMENT FACILITY (BMF)}

\subsection{The Bistatic Measurement Facility Specifications}

The Bistatic Measurement Facility is placed in an anechoic chamber $(2.70 \mathrm{~m} \times 2.10 \mathrm{~m})$. This facility enables the measurement of there flection factor, the magnitude and phase characteristics of the Device Under Test (DUT) under free space conditions. The target is placed in the geometrical center of the chamber and is protected with a flat absorber to avoid edge effects.

A high stable Anritsu vector network analyzer VNA (model 37269B) has been used as a generator of a continuous wave (CW) at X-band (from 9.4 to $11.7 \mathrm{GHz}$ ), with $230 \mathrm{MHz}$. Two corrugated horn antennas (transmitter/receiver), which are $1.2 \mathrm{~m}$ from the center, are pointed at the target by using a laser beam to avoid measurement errors associated and assume that their foot prints always overlap perfectly. Normally, the ideal case is when abroad-beam antenna is used for reception and a narrow-beam antenna for transmission. As in our BMF, the two antennas are almost the same and have identical 
footprints; therefore the focusing of the two antennas with a laser beam and a perfect mirror has to be done after each set of measurements. The antennas can be moved separately according to the incidence angle wanted and the sample can be moved up and down to correct for different thicknesses. An Agilent-VEE based software is used to move the antennas and to collect and to store the measured data from a network analyzer. Different tasks are possible with this program:

- Moving the two antennas either continuously with measurements each $0.4^{\circ}$ or discontinuously with measurements at steps of $1^{\circ}$.

- Turning the target to perform statistical (independent) measurements and adjust the target height. Indeed, for each setup, at least 40 independent samples have been measured.

- Set-up the wanted frequency and visualize the magnitude and phase of the reflectivity during the measurement.

The linear polarization of the antennas $(H$ or $V)$ can be chosen by changing manually the antenna dipole angles by $90^{\circ}$, so that the measurement of a full polarimetric scattering matrix is possible. The transmitter and the receiver are moving in the plane of incidence, where the azimuth angle of the transmitter is $0^{\circ}$ and the azimuth angle of the receiver is $180^{\circ}$ (Figure 1). The transmitter and the receiver can be moved from $12^{\circ}$ to $70^{\circ}$ simultaneously (specular case) or separately to measure the coherent and the incoherent term. However, due to mechanical problems, such as arm oscillations, the range of measurement is limited to $50^{\circ}$ or $60^{\circ}$, depending on the size of the target.

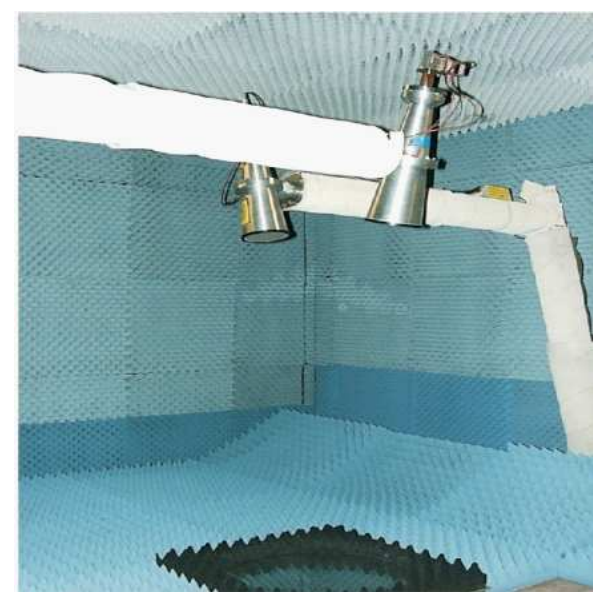

Figure 1. The bistatic measurement facility. 


\subsection{Soil Roughness}

The measurement of soil surfaces with known statistical properties of the roughness is relevant to understand and to validate the current theoretical models of scattering from soil and to analyze the effect of the roughness on the surface scattering. Two metallic stamps with different roughness have been constructed. These models can be used as a target or as a mould for shaping a target of selected soil materials with specified dielectric properties. The realization of the surface models needs two steps: as a first step, the surface height or the Digital Elevation Model (DEM) is generated as a data array for the wanted statistical parameters of the surface. Then, the metallic stamp (the surface model) is fabricated from $100 \times 100$ points array using a numerically controlled milling machine. The algorithm used for twodimensional DEM generation is described in reference, the spectral method used by Thorsos [12] and Boerner [13].

For this work, two DEM models have been generated, referred to as smooth for the small perturbation model (SPM), and rough for the physical optic model (PO). The generated surface models have been verified comparing their statistical properties calculated from the height array with the expected theoretical values; and we have found excellent agreements, for more details [40].

- Rough surface (PO): $k \sigma=0.515 ; k l=5.4$,

- Smooth surface (SPM): $k \sigma=0.1 ; m=0.1$.

where $\sigma$ the standard deviation of heights, $l$ the correlation length, $k$ the wave number and $m$ is the standard deviation of the slopes and $m=\sigma / l$ for a Gaussian surface. The rough and smooth surfaces chosen and the corresponding stamps are shown in Figures 2 and 3.

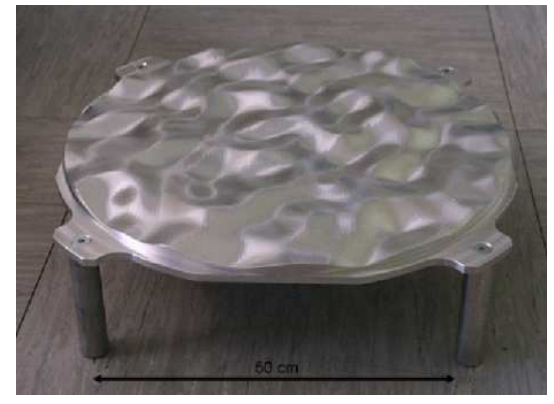

Figure 2. Rough surface, PO: $100 \times 100$ points array $(0.5 \times$ $0.5 \mathrm{~m})$.

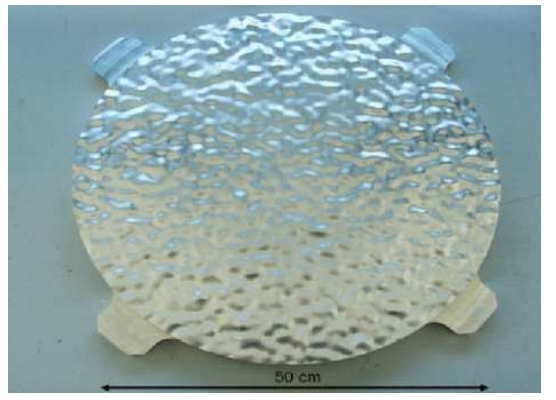

Figure 3. Smooth surface, SPM: $100 \times 100$ points array $(0.5 \times$ $0.5 \mathrm{~m})$. 


\subsection{Soil Moisture}

The measurement of soil moisture is one of the most important tasks of remote sensing and together with surface roughness, a very influential parameter for the surface scattering. Soil moisture is characterized by the amount of water held in a certain mass or volume of soil, therefore, the quantity of water in the soil can be described in two ways: the gravimetric quantity and the volumetric quantity [40]. Since the gravimetric method cannot be used for repetitive measurements at exactly the same position or the same target, the volumetric soil moisture will be measured by a Time Domain Reflectometry system (TDR). After the determination of the volumetric soil moisture, the dielectric constant of the soil can be derived using some empirical or semi-empirical model: the Topp model, the Dobson-Peplinsky Model [14,15], and the Hallikainen model [16]. From the expressions of these three models [40], the knowledge of the soil particle compositions is essential at least for the two last models. We were able to achieve a mechanical fractionation and sedimentation for three samples of soil to determine the sand and clay percentage contents of the material we used throughout our experiment, (see Table 1). An experimental measurement proves that the soil moisture remains almost the same after 10 hours, Figure 4.

Table 1. Soil particle compositions.

\begin{tabular}{|c|c|c|c|c|}
\hline $\begin{array}{c}\text { Soil } \\
\text { Sedimentation }\end{array}$ & Sample 1 & Sample 2 & Sample 3 & Average \\
\hline Sand & $95.5 \%$ & $95.2 \%$ & $96 \%$ & $95.56 \%$ \\
\hline Slit & $4.5 \%$ & $4.8 \%$ & $4 \%$ & $4.43 \%$ \\
\hline Clay & 0 & 0 & 0 & 0 \\
\hline
\end{tabular}

\subsection{Calibration of the BMF}

The different error sources present in the scattering matrix measurements are presented by the distortion matrix model. The general distortion matrix model or the calibration error model, which relates the ideal scattering matrix of the sample under test to the scattering matrix measured by the VNA, is represented by four matrices [17]:

$$
[M]=[R] \cdot[S] \cdot[T]+[B]
$$

$[S]$ is the desired (unknown) quantity, which represents the sample under test (SUT). $[M]$ and $[B]$ are directly measurable quantities; the 


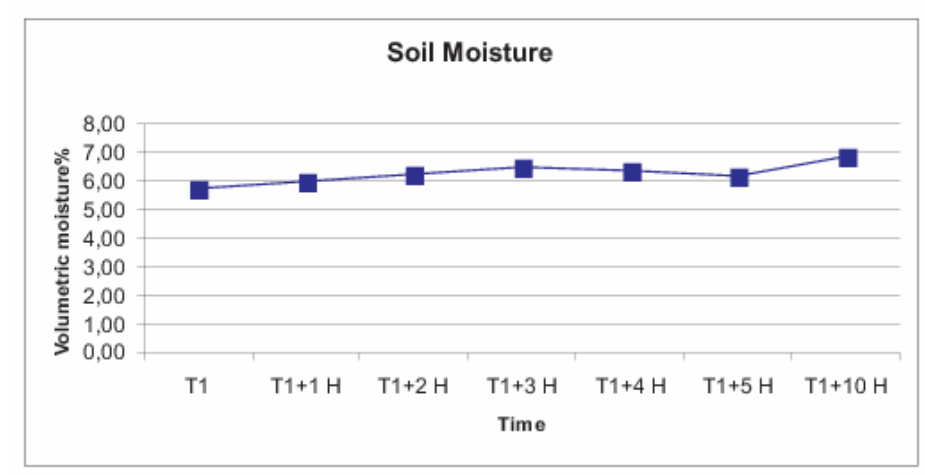

Figure 4. Time variation of the soil moisture.

first with the presence of the SUT and the second where the chamber is empty. $[R]$ and $[T]$ are respectively the receiver and the transmitter distortion matrix. Indeed, when $[S]=[0]$ then $[M]$ is equal to $[B]$. $[R]$ and $[T]$ are determined by using the calibration method. All of these are $2 \times 2$ complex matrices like the scattering matrix $[S]$ and they represent a 12 terms error model $[17,18]$.

The calibration process is achieved in 3 steps:

- measurement of $[M]$ and $[B]$ matrices,

- determination of $[R]$ and $[T]$ by comparing the measured matrix with the theoretical scattering matrix of a canonic target,

- determination of the scattering matrix by means of the following equation.

$$
[S]=[R]^{-1} \cdot([M]-[B]) \cdot[T]^{-1}
$$

The Isolated Antenna Calibration Technique (IACT), which has been used for our bistatic measurement facility, is detailed in $[19,20]$ and [21]. During the calibration process, different measurements were carried out using the metal plate to quantify the possible errors and to better understand the bistatic measurement facility. The metal plate should be polished enough to appear as a dull mirror, i.e., a reflected image of the anechoic room and equipment should be seen without any distortion. If the image is optically distorted, the radius of curvature will affect the $1 / R^{2}$ spreading of the $\mathrm{RF}$ wave. Therefore, the metal plate was reinforced with a metallic support to have a very good flatness. The co-polar terms, $S_{h h}$ and $S_{v v}$, of the theoretical scattering matrix of the metal plate have to be equal to 1 and the cross polar 


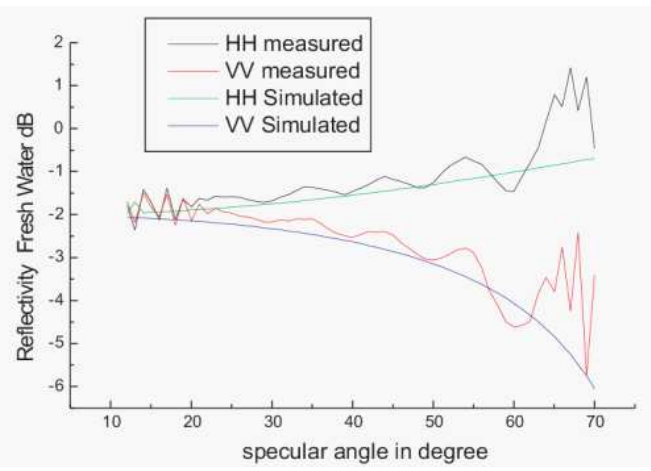

Figure 5. Validation of the calibration by means of a measurement of fresh water.

terms, $S_{h v}$ and $S_{v h}$, have to be equal to 0 . Therefore, the metal plate has to be big enough compared to the bistatic footprint. For example, it has to be at least 3 times the linear dimensions of the illumination spot. To validate the calibration process, the measurement of the fresh water reflectivity has been calibrated, corrected and then compared with the simulation. Since the reference target was a metal plate, which has a very high dielectric constant, it is recommended to validate the calibration with a dielectric target. Figure 5 shows that up to $50^{\circ}$ the maximum error is less than $0.5 \mathrm{~dB}$. In conclusion, a very well calibrated measurement could be carried out in the bistatic measurement facility using the IACT calibration process and the energy correction.

\section{VALIDATION OF THE BISTATIC SCATTERING COEFFICIENT OF ROUGH SURFACES WITH DIFFERENT SOIL MOISTURES AGAINST SIMULATED MODELS (IEM)}

To date, rigorous validation of the theoretical models have been carried out by using accurate numerical methods, i.e., methods of moments or Monte Carlo in a one-dimensional (1-D case) and with experimental data mostly collected on conducting surfaces. Evaluation of theoretical models by comparison with data collected on natural terrains is difficult, because of the difference between the theoretical autocorrelation functions used in the simulations and the real statistics of natural terrains. In this section, the experimental calibrated data are compared with simulation of the IEM method [22] to [26]. These data include a set of rough surface measurements with different soil moistures. 


\subsection{Calibrated Data for the Rough Surface (PO)}

For the rough surface, which is in the physical optics domain, measurements with four different soil moistures (5\% to 15\%) have been carried out and calibrated. The scattering matrix has been measured in the plane of incidence (vertical polarization plane) for different specular angles from $12^{\circ}$ to $47^{\circ}$ with steps of $5^{\circ}$. Figure 6 through Figure 9 show the specular angle variation for the different soil moistures and for the both polarizations $H H$ and $V V$. In Figure 5, one can see

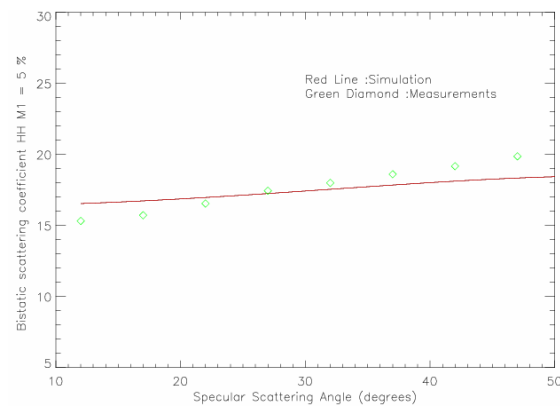

Figure 6. Calibrated coherent bistatic scattering coefficient vs. incidence angle, for the rough surface $(\mathrm{PO}), H H$ polarization and soil moisture: $M 1=5 \%$.

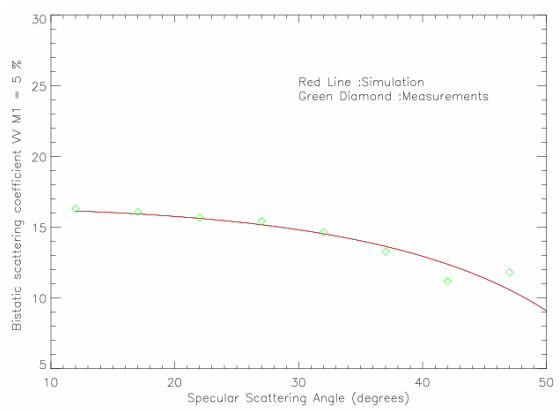

Figure 8. Calibrated coherent bistatic scattering coefficient vs. incidence angle for the rough surface $(\mathrm{PO}), V V$ polarization and soil moisture: $M 1=5 \%$.

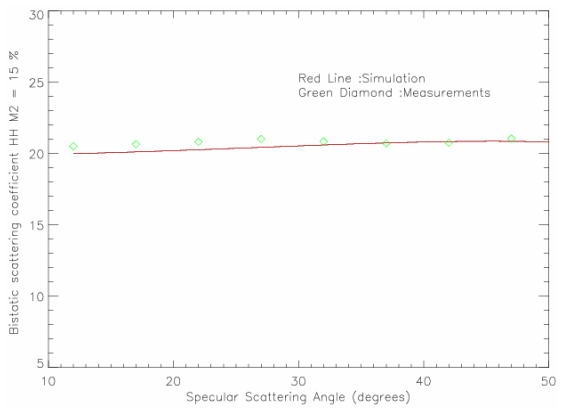

Figure 7. Calibrated coherent bistatic scattering coefficient vs. incidence angle for the rough surface $(\mathrm{PO}), H H$ polarization and soil moisture: $M 3=15 \%$.

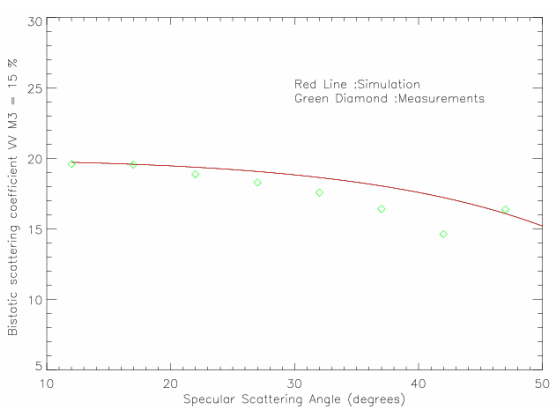

Figure 9. Calibrated coherent bistatic scattering coefficient vs. incidence angle for the rough surface $(\mathrm{PO}), V V$ polarization and soil moisture: $M 3=15 \%$. 
that the modelunder/overestimates the calibrated for $5 \%$ soil moisture. However, for the other three soil moistures the model fits very well to the calibrated data for the entire range of the specular angles.

On the other hand, for the $V V$ polarization, for the first three soil moistures a good agreement between the model and the calibrated data is obtained. For $15 \%$ soil moisture, the model tends to underestimate the calibrated data. This disagreement could be due to errors of the soil moisture measurement. Indeed, some transitive errors can be generated during the measuring process from the soil moisture to the dielectric constant.

\subsection{Calibrated Data for the Smooth Surface (SPM)}

For the smooth surface, which is in the small perturbation model domain, measurements with four different soil moistures (5\% to $15 \%$ ) have been carried out and calibrated. The scattering matrix has been measured in the plane of incidence (vertical polarization plane) for different specular angles from $12^{\circ}$ to $47^{\circ}$ with steps of $5^{\circ}$. As for the rough surface, we found a good agreement between the IEM model and the calibrated data. However, sometimes errors of $1 \mathrm{~dB}$ occur, which can be due to transitive errors of the dielectric constant measurements. These errors have to be considered during the surface parameters estimation.

\section{SOIL MOISTURE ESTIMATION VIA SPECULAR MEASUREMENTS}

The assessment of the soil moisture by radar remote sensing methods is possible by using the effect of the soil relative dielectric constant, which is related to the soil's moisture and the soil composition, on the received signal. Thus, a well-controlled measurement system and an accurate measured received signal enable the development of remote sensing techniques to evaluate the soil parameters. However, most of the developed techniques, which are specifically for the monostatic case, require different practical considerations and conditions. Indeed, the analytical models, which are used to asses either the soil moisture or the soil roughness by minimization of the metric distance between computed and measured data, are strongly nonlinear and are only defined for a limited range. By introducing multi-static or bistatic measurements, new perspectives or new techniques are possible. A new method, which requires data measured by radars operating along the specular direction, has been introduced by Ceraldi et al. [27]. This method, which has been confirmed until now only by method 
of moments (MoM) simulations, will be tested for the first time with accurately controlled measurements carried out in our anechoic chamber.

\subsection{Principles}

The measurement system parameters, such as the frequency or the polarization, could be a reliable tool to make the number of equations equal to the number of unknowns or to reduce the number of parameters to be estimated. Indeed, the measurement of the target with two different polarizations or frequencies could eliminate the dependence of the received power either on the roughness or on the relative dielectric constant. In fact, the different expressions of the scattered power of the analytical approximations (Kirchhoff or Small Perturbation) are a product of two functions: the first function is depending on the roughness and the second function is depending on the polarization and on the relative dielectric constant. Thus, the ratio of the scattered power in $H H$ to the scattered power in $V V$ is theoretically independent of the roughness [27],

$$
f(\theta, \varepsilon)=\frac{\sigma_{h h}^{0}}{\sigma_{v v}^{0}}=\frac{\left|R_{h}(\theta, \varepsilon)\right|^{2}}{\left|R_{v}(\theta, \varepsilon)\right|^{2}}
$$

Based on this underlying principle, assessment methods of the soil moisture using the copolarized ratio have been proposed by Shi et al. [28], and by Franceschetti et al. [29], but for the monostatic case only. However, those methods can only be used for slightly rough surfaces. Indeed, for very rough surfaces, where the geometrical optics model is valid, the sensitivity of the copolarized ratio to the relative dielectric constant is too small and it is equivalent to 1 . And for a medium rough surface where physical optics is valid, the copolarized ratio does not fit well with the physical optics approach. However, for bistatic scattering in the specular direction the copolarized ratio expression of the three different approaches, Small Perturbation Method (SPM), Physical Optics (PO) and Geometrical Optics (GO), are equal and independent of the roughness. Therefore, the estimation of the soil moisture by using the copolarized ratio is possible for a wider range of roughness in the specular direction. For more details see $[29,40]$.

\subsection{Results and Theory Validation}

The first validation of the theoretical expressions of the copolarized ratio, which is independent of the roughness, will be proved by using experimental calibrated measurement of two rough surfaces (smooth 


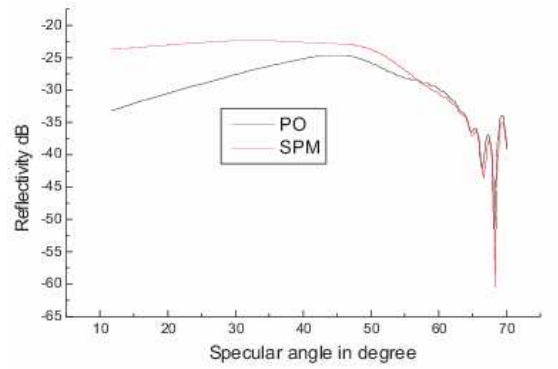

Figure 10. Reflectivity in the specular scattering direction for $H H$ polarization vs. incidence angle for the rough surface (PO), the smooth surface (SPM) and soil moisture: $M_{v}=5 \%$.

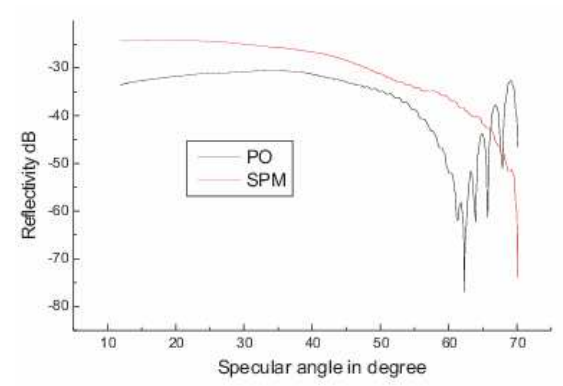

Figure 11. Reflectivity in the specular scattering direction for $V V$ polarization vs. incidence angle for the rough surface (PO), the smooth surface (SPM) and soil moisture: $M_{v}=5 \%$.

surface: SPM and rough surfaces: PO) with the same soil moisture. The validation was not possible for very rough surfaces: GO, because we were not able to construct a convenient sample stamp in our laboratory. Figure 10 shows the reflectivity of the smooth surface $(\mathrm{SPM})$ and of the rough surface (PO), with a constant soil moisture $\left(M_{v}=5 \%\right)$ for $H H$ polarization in the specular direction. One can clearly see that the reflectivity for the smooth surface is larger than for the rough surface. This is due to the diffuse part, which is proportional to the roughness. It has also to be noted that the reflectivity for the two roughnesses is almost the same for large specular angles. Figure 11, which is for $V V$ polarization, shows that the same remarks apply as for $H H$ polarization.

Figure 12 shows the copolarized ratio of the two different rough surfaces. Although the reflectivity is dependent on the roughness for the like polarizations $(H H$ and $V V)$, one can clearly see that the copolarized ratio is the same for the two rough surfaces. Based on the independence of the copolarized ratio of the roughness, which has been demonstrated by theory expressions and experimental calibrated measurements in the specular direction, an assessment algorithm, which evaluates the relative complex dielectric constant by the least squares technique, is proposed. Indeed, the estimated relative complex dielectric constant is the value which minimizes the sum of square modulus of the differences between the measured copolarized ratio and the corresponding analytic copolarized ratio. Thus, the measurement of the copolarized ratio at different incident angles (or frequencies) 


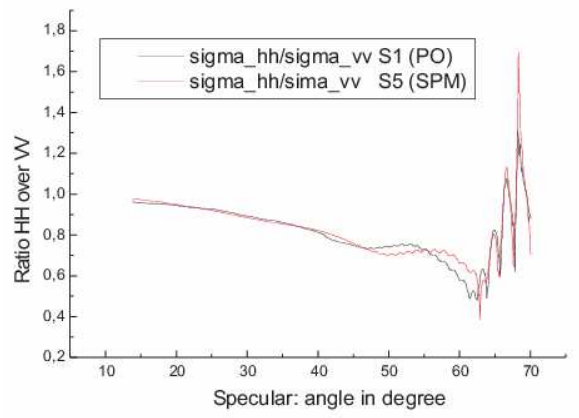

Figure 12. Copolarized ratio in the specular scattering direction vs. incidence angle for the rough surface (PO), the smooth surface (SPM) and soil moisture: $M_{v}=5 \%$. The copolarized ratio is independent of roughness.

allows us to estimate the complex relative dielectric constant by minimizing the following function [27],

$$
g\left(\varepsilon^{\prime}, \varepsilon^{\prime \prime}\right)=\sum_{i}\left|f\left(\varepsilon^{\prime}, \varepsilon^{\prime \prime}, \theta_{i}, \lambda_{i}\right)-\hat{f}\left(\theta_{i}, \lambda_{i}\right)\right|^{2}
$$

where $\varepsilon^{\prime}$ and $\varepsilon^{\prime \prime}$ are respectively the real and the imaginary part of the complex relative dielectric constant. $\hat{f}(\theta, \lambda)$ is the measured copolarized ratio and $f\left(\varepsilon^{\prime}, \varepsilon^{\prime \prime}, \theta, \lambda\right)$ is the analytic copolarized ratio. To analyze the performance of the previous algorithm, a set of well controlled polarimetric measurements have been carried out. For both surface roughness values, the rough surface (PO) and the smooth surface (SPM), measurements with four soil moistures $M_{v}(5 \%, 10 \%$, $15 \%$ and $20 \%$ ) have been carried out for different specular angles varying from $12^{\circ}$ to $52^{\circ}$ with steps of $5^{\circ}$. To improve the quality of the data, the average of the statistical measurements which have been subsequently calibrated, have been used.

Figures 13 and 14 show the estimated real part and the imaginary part of the relative dielectric constant, respectively, versus the incidence angle for the rough surface (PO) and for the different soil moistures. The purpose of these plots is to find out the best angle to estimate the relative dielectric constant. In fact, one can see that a good agreement between the estimated values and the measured values is obtained for large specular angle. It turns out that from the specular angle of $32^{\circ}$ the estimated real part of the relative dielectric constant shows best agreement. Similarly, the estimated imaginary parts of the relative dielectric constant are acceptable for the specular angle of $32^{\circ}$.

Figures 15 and 16 show the estimated real and imaginary part 


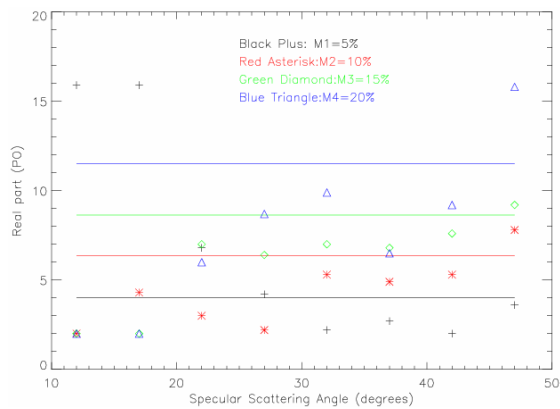

Figure 13. Estimated real part of the relative dielectric constant vs. incidence angle for the rough surface (PO). Lines: measured values.

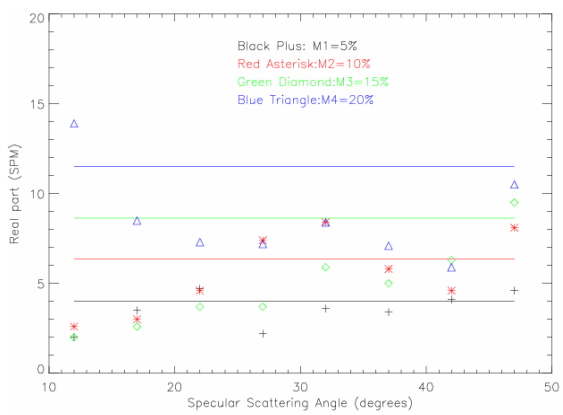

Figure 15. Estimated real part of the relative dielectric constant vs. incidence angle for the smooth surface (SPM). Lines: measured values.

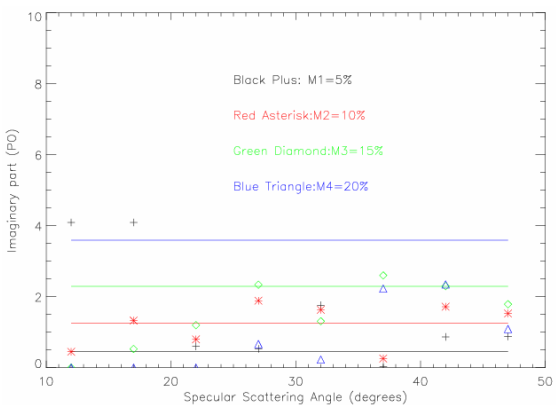

Figure 14. Estimated imaginary part of the relative dielectric constant vs. incidence angle for the rough surface (PO). Lines: measured values.

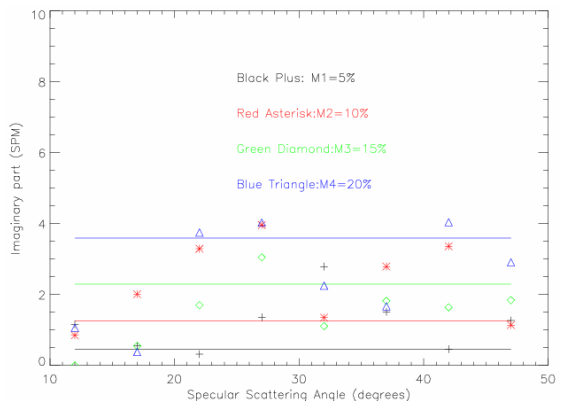

Figure 16. Estimated imaginary part of the relative dielectric constant vs. incidence angle for the smooth surface (SPM). Lines: measured values.

of the relative dielectric constant, respectively, versus the incidence angle for the smooth surface (SPM) and for the different soil moistures. Similar to the rough surface, the best agreement between the estimated values and the measured values is again obtained for the large specular angle (from the specular angle of $32^{\circ}$. However, some good results can be observed for small specular angles, in agreement with the theory. Indeed, in [28] and [29], an algorithm based on the copolarized ratio, which has been used for monostatic radar configuration, showed good results only for slightly rough surfaces. Further, for smooth surfaces, the proposed algorithm can be used for the quasi-monostatic case (low 
bistatic angles).

The correlation between the estimated and measured relative dielectric constant for four specular angles $\left(32^{\circ}, 37^{\circ}, 42^{\circ}\right.$ and $\left.47^{\circ}\right)$ is presented in Figures 17, 18, 19 and 20. From these figures, one can say that the accuracy of this algorithm is acceptable for different applications. A high decorrelation between the estimated and measured dielectric constant, may be caused by calibration errors or by the miss-estimation of the real value of the measured dielectric constant.

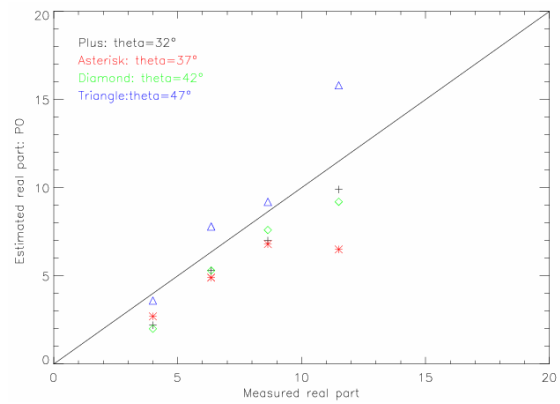

Figure 17. Estimated real part of the relative dielectric constant vs. measured relative dielectric constant for the rough surface $(\mathrm{PO})$.

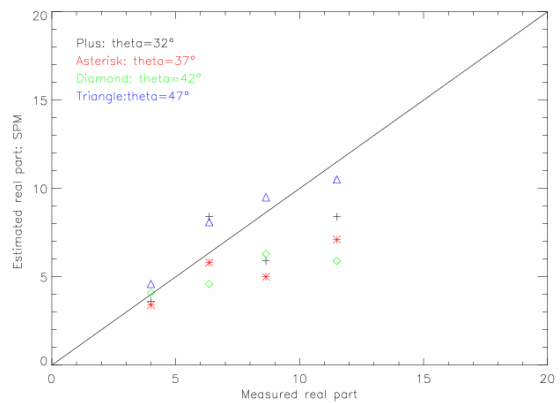

Figure 19. Estimated real part of the relative dielectric constant vs. measured relative dielectric constant for the smooth surface (SPM).

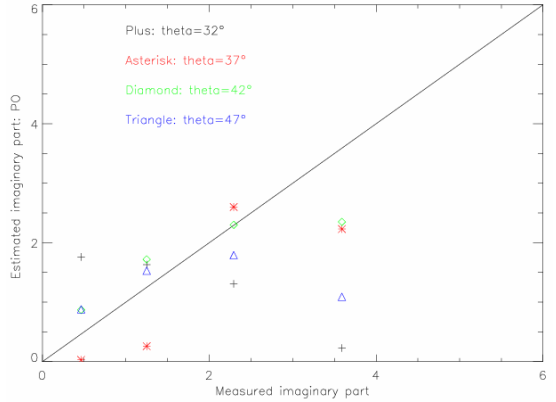

Figure 18. Estimated imaginary part of the relative dielectric constant vs. measured relative dielectric constant for the rough surface (PO).

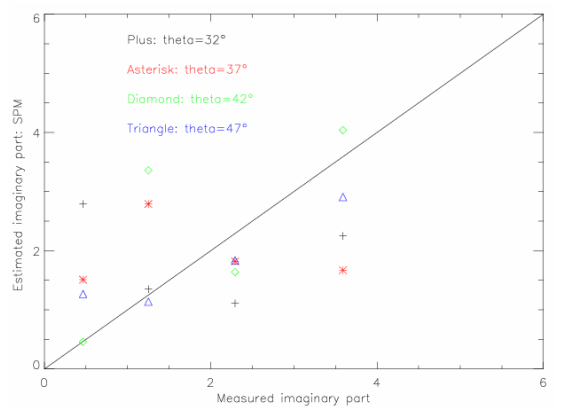

Figure 20. Estimated imaginary part of the relative dielectric constant vs. measured relative dielectric constant for the smooth surface (SPM). 


\section{ROUGHNESS ESTIMATION VIA SPECULAR MEASUREMENTS}

The coherent term of the integral equation method is a function of the dielectric constant (or the soil moisture) and the soil roughness $\sigma$ (the standard deviation of heights) [23, 24]:

$$
\mathrm{IEM}_{c o h}=f(\varepsilon, \sigma)
$$

Thus, if the dielectric constant is known, the soil roughness $\sigma$ can be deduced from the coherent IEM term. The IEM approximation is valid for a wide range of roughness values; therefore the assessment of $\sigma$ from the coherent IEM term could be a reliable tool. As the average of the incoherent part is zero, the coherent part can be calculated by the average of a set of statistical data. It has tobe noted that, the coherent part has to be at least comparable to the incoherent part to have acceptable results. Indeed, for a too low coherent part, i.e., very high roughness, the IEM does not fit the measured coherent part very well [23] and [25].

In Figures 21 and 22, the IEM coherent and incoherent scattering coefficient versus the roughness, for the $V V$ polarizations and for different soil moistures are shown. One can clearly see that up to $k \sigma$ equal to 1.5 , the coherent part is comparable to the incoherent term and thus the assessment of the roughness $\sigma$ from the coherent part is possible. As expected, for very high roughness the coherent part is too low. And for very high roughness, the IEM does not fit the measured

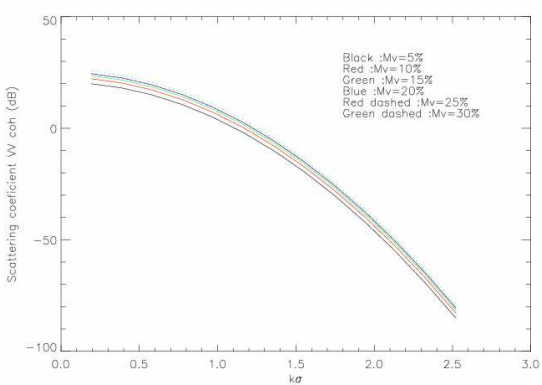

Figure 21. Coherent integral equation method: Scattering coefficient for the specular angle $20^{\circ}$ and for $V V$ polarization vs. spectral roughness $k \sigma$ for a Gaussian surface: $l=0.73$ and soil moisture: $M_{v}$ varies from $5 \%$ to $30 \%$.

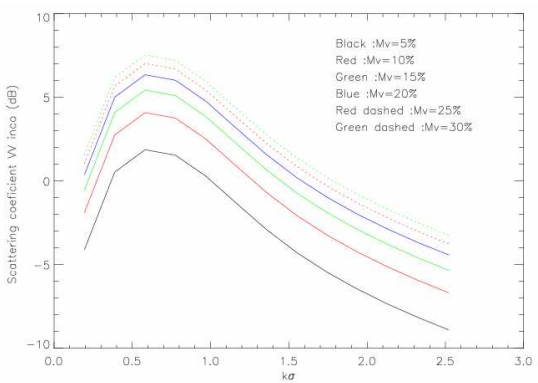

Figure 22. Incoherent integral equation method: Scattering coefficient for the specular angle $20^{\circ}$ and for $V V$ polarization vs. spectral roughness $k \sigma$ for a Gaussian surface: $l=0.73$ and soil moisture: $M_{v}$ varies from $5 \%$ to $30 \%$. 
Table 2. Estimated spectral roughness $k \sigma$ for different specular angles and soil moisture, using the $H H$ IEM coherent (rough surface: PO).

\begin{tabular}{|c|c|c|c|c|}
\hline $\begin{array}{c}\mathrm{PO}, H H: \\
k \sigma=0.51\end{array}$ & $32^{\circ}$ & $37^{\circ}$ & $42^{\circ}$ & $47^{\circ}$ \\
\hline$M 1=5 \%$ & 0.41 & 0.18 & 0.43 & 0.29 \\
\hline$M 2=10 \%$ & 0.46 & 0.4 & 0.49 & 0.63 \\
\hline$M 3=15 \%$ & 0.46 & 0.52 & 0.57 & 0.64 \\
\hline$M 4=20 \%$ & 0.56 & 0.51 & 0.67 & 0.84 \\
\hline
\end{tabular}

Table 3. Estimated spectral roughness $k \sigma$ for different specular angles and soil moisture, using the $V V$ IEM coherent (rough surface: PO).

\begin{tabular}{|c|c|c|c|c|}
\hline $\begin{array}{c}\mathrm{PO}, V V: \\
k \sigma=0.51\end{array}$ & $32^{\circ}$ & $37^{\circ}$ & $42^{\circ}$ & $47^{\circ}$ \\
\hline$M 1=5 \%$ & 0.61 & 0.58 & 0.72 & 0.98 \\
\hline$M 2=10 \%$ & 0.64 & 0.69 & 0.90 & 0.97 \\
\hline$M 3=15 \%$ & 0.65 & 0.77 & 0.95 & 0.96 \\
\hline$M 4=20 \%$ & 0.72 & 0.76 & 0.98 & 1.04 \\
\hline
\end{tabular}

coherent part very well. We have got the same conclusions for the $H H$ polarization.

In Tables 2 and 3, the results obtained for the rough surface (PO: $k \sigma=0.51$ ) and for the $H H$ and $V V$ polarization respectively are shown. For the $H H$ polarization, good agreement between the estimated and the measured spectral roughness $k \sigma$ is obtained for the first three specular angles, $32^{\circ}, 37^{\circ}$ and $42^{\circ}$. For the specular angle $47^{\circ}$, the estimated values underestimated the measured $k \sigma$. For the $V V$ polarization, good results are only obtained for the specular angles $32^{\circ}$ and $37^{\circ}$.

\section{PHASE SENSITIVENESS TO SOIL MOISTURE IN SPECULAR DIRECTION}

In the last two decades, SAR Interferometry (InSAR) and differential InSAR (DInSAR), which use the phase of the backscattered signal, have been shown to be a useful tool for the generation of Digital Elevation Models (DEMs), and the study of temporal changes due to earthquakes, subsidence, and other ground motions. Nolan [3032 , also suggested the possibility to use DInSAR penetration depth 
as a proxy to estimate the soil moisture. The principle is based on the relationship between penetration depth and permittivity, which varies as a function of soil moisture. In this section, we will study the sensitivity of signal phase and reflectivity with regard to moisture variation and therefore to penetration depth. Current results indicate a non-linear relationship between signal phase and soil moisture, as expected, confirming the possibility of using DInSAR to measure variations in soil moisture.

For the monostatic geometry, due to the randomness of the volume scattering in soil, the scattered wave is diffused in all directions and its signal phase changes in a random way with each scattering on a discontinuity of the medium. Nevertheless in the specular case, the wave undergoes with a greater probability coherent scattering on the surface, where the dielectric contrast is too high [33]. For the coherent scattering, the phase shift is deterministic and it is given by the formula of coherent scattering on laminated mediums approximated by the Wentzel-Kramer-Brillouin (WKB) model [34]. Outside of the specular direction, the random fluctuations of the phase are increased by the effect of the volume scattering: this effect is valid also for a smooth plane surfaces and rough surface smaller than the macroscopic roughness. In the monostatic geometry, the condition of specularity is satisfied only for normal incidence. We thus explain the increase in the phase shift decorrelation with the value of the incidence angle in the case of the monostatic geometry. Therefore in this section, the signal phase sensitivity to soil moisture for the specular direction will be reported.

\subsection{Theory}

On reflection at a surface, an electromagnetic wave undergoes a phase shift. The value of this shift is equal to the phase of the Fresnel complex reflection coefficient. The relative phase shift is defined as the difference of the phase for samples which differ only by their dielectric properties (soil moisture). In the case of homogeneous media, the phase jump on the surface is given by the formula of Fresnel [33],

$$
\varphi=\arg \left[\frac{1-\sqrt{\varepsilon^{\prime}-i \varepsilon^{\prime \prime}}}{1+\sqrt{\varepsilon^{\prime}-i \varepsilon^{\prime \prime}}}\right]
$$

The reflection coefficient of soil depends on all the values of complex dielectric constant in the upper level of the soil with thickness equal to the penetration depth. Thus, the penetration depth plays a fundamental role in the surface scattering process (reflectivity and phase). For homogeneous media, the Fresnel coefficient can be useful for the interferometric phase calculation [35]. However, it is necessary 
for the calculation of the interferometric phase to consider not only the soil moisture but also gradients of the water contents in the soil, considering the very large sensitivity of the phase to the values of these gradients. Thus, to analyze the signal phase of a wave scattered by a smooth surface, the method of the layers [34], allows the exact calculation of the phase, which depends on all the values of the complex dielectric constant up to the penetration depth.

The remote sensing observation depth is defined as the depth of soil where the soil moisture can be estimated or where the microwave radar is sufficiently sensitive. Thus, the radar observation depth is a function of the soil moisture and it is greater for dryer soil than for moist soil. As well as the soil moisture, the remote sensing observation depth is also depending on free space wavelength, incidence angle, wave polarization, surface roughness and vegetation cover [36-38]. The radar penetration depth $\delta_{p}$, which has been introduced by Ulaby et al. [39], is a function of the radar frequency system and soil moisture (soil dielectric constant). Indeed, the radar penetration depth is the depth in the soil where the transmitted wave power is attenuated by the proportion $1 / e$ (i.e., $37 \%$ ) of the incident wave power and without considering the scattered power wave on the soil surface [39],

$$
\delta_{p}=\frac{\lambda \sqrt{\varepsilon^{\prime}}}{2 \pi \varepsilon^{\prime \prime}}
$$

where $\lambda$ is the free space wavelength, $\varepsilon^{\prime}$ is the real part of the soil relative dielectric constant and $\varepsilon^{\prime \prime}$ is the imaginary part of the soil relative dielectric constant. Contrary to the radar observation depth, the radar penetration depth could be a reliable proxy to assess the soil moisture by measuring the signal phase of the received wave. Indeed, the moisture variation (penetration depth variation) causes a change in path length and yields a change in phase, the so-called signal phase [32],

$$
\phi_{\text {sig }}=\delta_{p} \frac{4 \pi}{\lambda}
$$

However, we have to remember that the path change of the signal is a virtual distance variation, which depends on the amount of energy penetrating the surface and on that scattered back to the receiver.

The Hallikainen model [16], relates the dielectric constant to the frequency, the volumetric moisture content and the percentage of sand and clay contained in the soil. It has been used to prove the relationship between the soil moisture and penetration depth [16],

$$
\varepsilon_{c}=\left(a_{0}+a_{1} S+a_{2}\right)+\left(b_{0}+b_{1}+b_{2} C\right) m_{v}+\left(c_{0}+c_{1} S+c_{2} C\right) m_{v}^{2}
$$

$\varepsilon_{c}$ is the complex dielectric constant, $S$ is the percentage of sand, $C$ is the percentage of clay, $m_{v}$ is the volumetric moisture content, and the coefficients $a_{i}, b_{i}$ and $c_{i}$ depend on the frequency. 


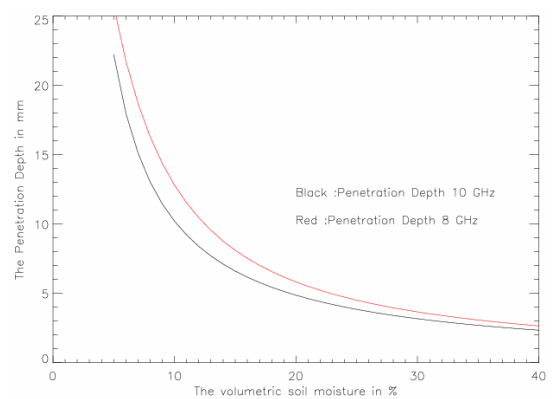

Figure 23. Penetration depth versus volumetric soil moisture.

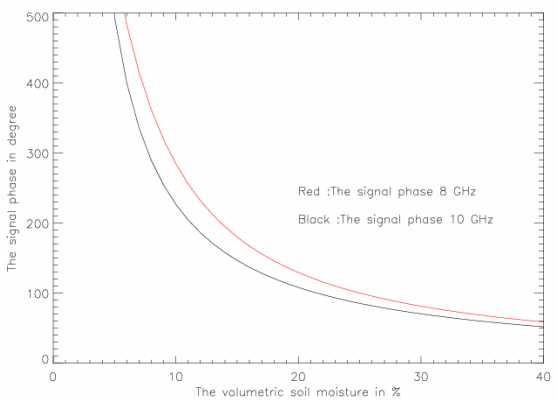

Figure 24. Signal phase versus volumetric soil moisture.

Because of the non-linear relationship between soil moisture and penetration depth, (see Figure 23), a measurement of a change in penetration depth cannot be directly converted to a change in soil moisture unless one of the soil moisture values is known a priori or if some linearizing assumptions are made. For example, a measured displacement of $5 \mathrm{~mm}$ could ambiguously mean a change in soil moisture from $1-2 \%$ or from $10-17 \%$. However, if the initial soil moisture value is known, and assuming that a phase change is fully attributable to a change in penetration, the initial value can be converted to a penetration depth using the equations previously presented.

Figure 24 shows the variation of the signal phase versus soil moisture. Due to the linear relationship between the signal phase and the penetration depth, (7), the signal phase is also non-linearly related to the soil moisture.

\subsection{Experimental Lab Measurements and Results}

The chosen target was a flat soil with different moisture levels controlled by a TDR measurement system. An average of four soil moisture measurements was used for each target. The soil sedimentation shows that the soil contains $100 \%$ (pure) sand and no clay contributions. Therefore, the swelling effect of soil caused by clay can be completely neglected.

Specular measurements with different soil moistures have been carried out to investigate the signal phase variation with the soil moisture, as the penetration depth is related to the magnitude of the reflected signal. The variation of reflectivity with soil moisture is shown in Figure 25; the incidence angle was of 20 degree. 


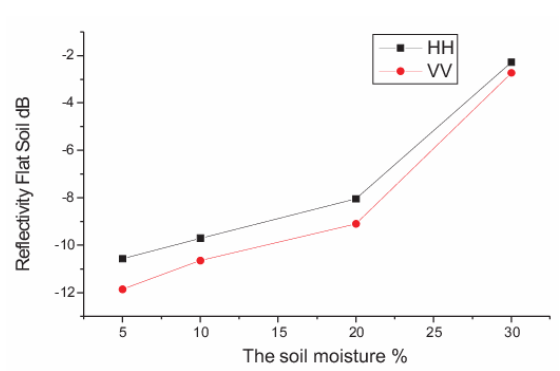

Figure 25. Reflectivity of flat soil versus soil moisture.

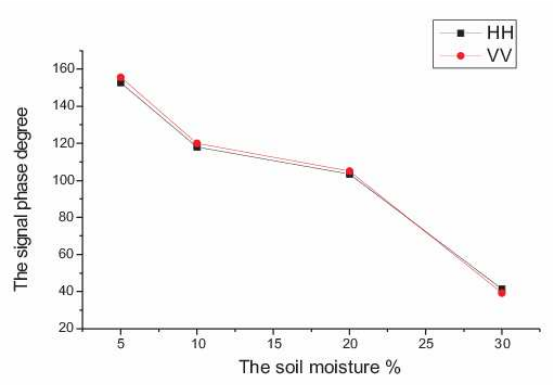

Figure 26. Signal phase versus the soil moisture.

The reflectivity of flat soil increases as the soil moisture increases for both polarizations $H$ and $V$. Apparently the penetration depth depends on the soil moisture. Figure 26 shows that the signal phase is also changing with the soil moisture (for the same geometry and the same soil roughness).

Figure 27 shows that the maximum variation of the phase shift using the Fresnel reflection coefficient, (Equation (9)), is about 2 degree for soil moistures varying from $5 \%$ to $40 \%$ (sandy soil). This means that the measured variation of the signal phase of up to $100^{\circ}$ cannot be explained by the pure dielectric effect covered by the Fresnel equations.

The assessment and interpretation of the signal phase for the different soil moistures using the complex interferometric coherence seems to be reasonable. The coherence is defined as follows [32],

$$
\Gamma=\frac{\left\langle S_{i} S_{r}^{*}\right\rangle}{\sqrt{\left\langle\left|S_{i}\right|^{2}\left|S_{r}\right|^{2}\right\rangle}}
$$

where $S_{r}$ is the reference signal of a surface with soil moisture $m_{v 1} . S_{i}$ with $i=2,3$ is the complex amplitude of the signal for the surfaces with soil moistures $m_{v i}$. \langle\rangle denotes ensemble averaging.

As expected, due to the coherent nature of the surface (flat soil), the magnitude of the interferometric coherence for two relatively wet surfaces is almost 1 . However, the main purpose of this experiment was the analysis of the signal phase measured for different soil moistures at the same geometry and roughness. Therefore, only the argument of the complex coherence, i.e., the phase shift due to the soil moisture variation, will be taken into account. From Figure 28, one can see that this phase shift can be a good proxy to assess the soil moisture variation. Due to the absence of clay in our soil (no swelling effects), the phase shift can be directly related to the penetration depth of the electromagnetic wave into the soil. 


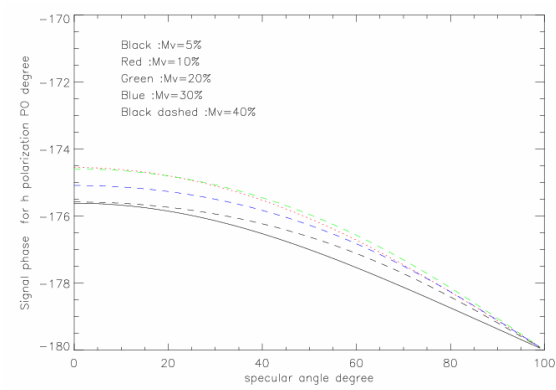

Figure 27. Signal phase versus the soil moisture.

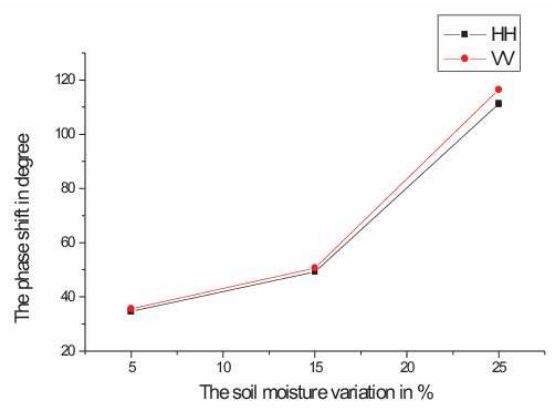

Figure 28. Interferometric phase versus soil moisture variation.

The variation of the reflectivity with respect to the soil moisture could be a reliable tool to understand the relationship between the penetration depth and soil moisture. As expected, it can be seen from the measurements that the penetration depth decreases within creasing reflectivity. The dependence of the signal phase on the soil moisture demonstrates that the path of the electromagnetic wave through the soil is strongly related to its dielectric properties. The nonlinearity of the signal phase variation to the soil moisture variation can be clearly seen, but the biggest changes in phase are occurring at the higher ranges of soil moisture which is contrary to theory. It could be a problem of correct phase unwrapping (i.e., cycle slips), but it may be also due to inaccuracies in the measurements. The effects of surface roughness for varying bistatic angles and polarization should be the subject of future studies. The final aim is to find ways to independently estimate soil moisture and surface roughness.

\section{CONCLUSION}

The X-band Bistatic Measurement Facility (BMF) at the DLR Oberpfaffenhofen, Microwaves and Radar Institute has been detailed. The BMF has been calibrated using the Isolated Antenna Calibration Technique (IACT). And the validation of the calibration was achieved by measuring the reflectivity of fresh water. Measurement of the bistatic scattering coefficient with accuracy better than $0.5 \mathrm{~dB}$ could be achieved. Bistatic measurement sets of soils with different statistical roughness and different moistures controlled by a TDR system have been carried out. The calibrated data have been validated against the IEM model. We have found good agreements between the IEM model and the calibrated data. Finally, we can say that these well 
calibrated data are reliable tools to investigate the methods for the surface parameters estimation, which is the main task of the paper.

By using the calibrated data measured in the specular direction, it could be verified that the co-polarized ratio of the scattering coefficient is independent of the soil roughness, which is in agreement with the theory. For both the roughness surfaces and for the real and imaginary part of the dielectric constant, the best estimated values have been found for large specular angles starting from $32^{\circ}$. By knowing the dielectric constant from the specular algorithm, the vertical roughness can be calculated from the expression for the coherent part of IEM. This new idea gave us very good results as a first validation. However, sometimes the correlation between the estimated and the measured roughness is too low. We think that it is due to the transitive errors resulting from the specular algorithm. The sensitivity of signal phase to soil moisture, which has a coherent behavior in the specular direction, has been reported. Current results confirm the possibility of using the signal phase, which can be evaluated from differential interferometry techniques, to estimate the soil moisture.

The experiments and analysis show that important surface parameters, such as soil moisture and roughness, can be measured independently using a bistatic, fully polarimetric X-band radar operating close to a specular geometry. Although, such geometries have the disadvantage of lower range resolution than monostatic systems with no resolution at the specular angle, useful resolution can be achieved close to specular with the advantage of greater sensitivity, due to the higher reflectivity from forward scattering. Bistatic, fully polarimetric remote sensing systems using pairs of SAR satellites, notably TanDEM-X are planned for the near future [1]. Although their primary purpose is to measure the Earth's topography using SAR interferometry and the geometry is close to monostatic, TanDEM-X demonstrates that the requirements of the techniques described in this paper are feasible even from space.

\section{ACKNOWLEDGMENT}

The authors would like to thank Mr. Daniel Glaser, chemical technician of the Technical University of Munich, for the sedimentation of the soil used in this experiment.

\section{REFERENCES}

1. Krieger, G., A. Moreira, H. Fiedler, I. Hajnsek, M. Werner, M. Younis, and M. Zink, "TanDEM-X: A satellite formation 
for high-resolution SAR interferometry," IEEE Trans. Geosci. Remote Sensing, Vol. 45, No. 11, 3317-334, Nov. 2007.

2. Oh, Y., K. Sarabandi, and F. T. Ulaby, "An empirical model and aninversion technique for radar scattering from bare soil surfaces," IEEE Trans. Geosci. Remote Sensing, Vol. 30, No. 2, 370-381, 1992.

3. Dubois, P. C., J. J. van Zyl, and T. Engman, "Bistatic scattering and emissivities of lossy dielectric surfaces with exponential correlation functions," IEEE Trans. Geosci. Remote Sensing, Vol. 33, No. 4, 916-926, 1995.

4. $\mathrm{Xu}, \mathrm{P}$. and L. Tsnag, "Measuring soil moisture with imaging radars," IEEE Trans. Geosci. Remote Sensing, Vol. 45, No. 1, Jan. 2007.

5. Wu, T. D., K. S. Chen, J. Shi, H. W. Lee, and A. K. Fung, "A study of an AIEM model for bistatic scattering from randomly rough surfaces," IEEE Trans. Geosci. Remote Sensing, Vol. 46, No. 9, Sep. 2008.

6. Cost, S. T., "Measurements of the bistatic echo area of terrain of X-band," Ohio State University, Antenna Lab., Rept. 1822-2, May 1965.

7. Domville, A. R., "The bistatic reflection from land and sea of Xband radio waves," pt. I, GEC (Electronics) Ltd., Memo. SLM 1802, Stanmore, England, Jul. 1967.

8. Domville, A. R., "The bistatic reflection from land and sea of Xband radio waves," pt. II, GEC (Electronics) Ltd., Memo. SLM 2116, Stanmore, England, Jul. 1968.

9. Domville, A. R., "The bistatic reflection from land and sea of Xband radio waves," pt. II Suppl., GEC-AEI (Electronics) Ltd., Memo. SLM 2116 (Suppl.), Stanmore, England, Jul. 1969.

10. De Roo, R. D., "Theory and measurement of bistatic scattering of X-band microwaves from rough dielectric surfaces," Ph.D. Thesis, Michigan University, USA, 1996.

11. Macelloni, G., G. Nesti, P. Pampaloni, S. Sigismmondi, D. Tarchi, and S. Lolli, "Experimental validation of surface scattering and emission models," IEEE Trans. Geosci. Remote Sensing, Vol. 38, No. 1, Jan. 2000.

12. Thorsos, E. I., "The validity of the Kirchhoff approximation for rough surface scattering using a Gaussian roughness spectrum," Journal Acoust. Soc. AM., Vol. 83, No. 1, 78-92, Jan. 1988.

13. Boerner, T., "Development of a coherent scattering model for polarimetric SAR interferometry applications," Ph.D. Thesis, 
Munich University, Germany, 2000.

14. Dobson, G. C., J. L. Davis, and A. P. Annan, "Electromagnetic determination of soil water content: Measurements in coaxial transmission lines," Water Resour. Res., Vol. 16, No. 3, 574-582, 1980.

15. Dobson, M. C., F. T. Ulaby, M. T. Hallikainen, and M. El-Rayes, "Microwave dielectric behaviour of wet soil - Part II: Dielectric mixing models," IEEE Trans. Geosci. Remote Sensing, Vol. 23.1, 35-46, 1985.

16. Hallikainen, M. T., M. C. Ulaby, M. C. Dobson, and M. El-Rayes, "Microwave dielectricbehavior of wet soil - Part I: Empirical models and experimental observations," IEEE Trans. Geosci. Remote Sensing, Vol. 23.1, 25-34, 1985.

17. Wiesbeck, W., "A complete error model for polarimetric RCS- and antenna measurements," Institut fuer Hoechstfrequentechnik und Elektronik, University Karlsruhe, Kaiserstr, 127500 Karlsruhe, Federal Republic of Germany, 1992.

18. Jersak, B. D., "Bistatic, fully polarimetric radar cross-section calibration techniques and measurement error analysis," Ph.D. Thesis, The University of Texas at Arlington, 1993.

19. Sarabandi, K. and F. Ulaby, "A convenient technique for polarimetric calibration of single-antenna radar systems," IEEE Trans. Geosci. Remote Sensing, Vol. 28, No. 6, 1022-1033, Nov. 1990.

20. Sarabandi, K., F. T. Ulaby, and M. A. Tassoudji, "Calibration of polarimetric radarsystems with good polarization isolation," IEEE Trans. Geosci. Remote Sensing, Vol. 28, No. 1, 70-75, Jan. 1990.

21. Hauck, B., F. T. Ulaby, and R. DeRoo, "Polarimetric bistaticmeasurement facility for point and distributed targets," IEEE Transaction on Antennas and Propagations, Vol. 40, No. 1, Feb. 1998.

22. Fung, A. K. and G. W. Pan, "Backscattering from a randomly rough dielectric surface," IEEE Trans. Geosci. Remote Sensing, Vol. 30, No. 2, Mar. 1992.

23. Koufogbo, F., "Modelling of rough natural and manmade surfaces at millimetre-wave frequencies - Study of the interactions between a target and its natural environment," Ph.D. Thesis, The Paul Sabatier University, Toulouse, France, 2002.

24. Tsang, L., J. A. Kong, and R. T. Shin, Theory of Microwave Remote Sensing, Wiley-Interscience Publication, 1985. 
25. Alvarez, J. L., "Two novel studies of electromagnetic scattering in randommedia in the context of radar remote sensing," Ph.D. Thesis, University of Nottingham, UK, Mar. 2002.

26. Alvarez, J. L., "An extension of the IEM/IEMM surface scattering model," Waves Random Media, Vol. 11, Mar. 2001.

27. Ceraldi, E., G. Franceschetti, A. Iodice, and D. Riccio, "Estimating the soil dielectric constant via scattering measurements along the specular direction," IEEE Trans. Geosci. Remote Sensing, Vol. 43, No. 2, 295-305, Feb. 2005.

28. Shi, J., J. Wang, A. Y. Hsu, P. E. O'Neill, and E. Engman, "Estimation ofbare surface soil moisture and surface roughness parameter using L-band SAR image data," IEEE Trans. Geosci. Remote Sensing, Vol. 35, No. 5, 1254-1266, Sep. 1997.

29. Franceschetti, G., A. Iodice, S. Maddaluno, and D. Riccio, "A fractal based theoretical framework for the retrieval of surface parameters from electromagnetic backscattering data," IEEE Trans. Geosci. Remote Sensing, Vol. 38, No. 2, 641-650, Sep. 2000.

30. Nolan, M., D. R. Fatland, and L. Hinzman, "DInSAR measurements of soil moisture," IEEE Trans. Geosci. Remote Sensing, Vol. 41, No. 12, Dec. 2003.

31. Nolan, M. and D. R. Fatland, "New DEMs may stimulate significant advancements in remote sensing of soil moisture," EOS Trans. AGU, Vol. 84, No. 25, 233-240, Jun. 24, 2003.

32. Nolan, M. and D. R. Fatland, "Penetration depth as a DInSAR observable and proxy for soil moisture," IEEE Trans. Geosci. Remote Sensing, Vol. 41, No. 3, 532-537, Mar. 2003.

33. Despan, D., "Modles thoriques et mesures de la diffusion du rayonnement lectromagntique dans le domaine optique et radar par des sol avec dessurfaces lisses et rugueuses alatoires," Ph.D. Thesis, Universit de Marne-la-Valle, Feb. 1999.

34. Wait, J. R., Electromagnetic Waves in Stratified Media, Pergamon Press, Oxford, 1996.

35. Rudant, J. R., A. Bedidi, D. Massonet, G. Nesti, and R. Calonne, "Decorrelation of back scattered signal due to soil moisture changes," Conference CNES, Toulouse, Oct. 1995.

36. Arya, L. M., J. C. Richter, and J. F. Paris, "Estimating profile waterstorage from surface zone soil moisture measurements under bare field conditions," Water Resour. Res., Vol. 19, No. 2, 403$412,1983$.

37. Rudant, J. R., A. Bedidi, D. Massonet, G. Nesti, and R. Calonne, "Near surface soil moisture estimation from 
microwave measurements," Remote Sens. Environ., Vol. 26, 101121, Oct. 1988.

38. Engman, E. T. and N. Chauhan, "Status of microwave soil moisture measurements with remote sensing," Remote Sens. Environ., Vol. 51, No. 1, 189-198, 1995.

39. Ulaby, F. T., R. K. Moore, and A. K. Fung, Microwave Remote Sensing: Active and Passive, Vol. II - Radar Remote Sensing and Surface Scattering and Emission Theory, 609, AddisonWesley, Advanced Book Program, Reading, Massachusetts, 1982.

40. Ben Khadhra, K., "Surface parameter estimation using bistatic polarimetric X-band measurements," Ph.D. Thesis, German Aerospace Centre (DLR), Microwaves and Radar Institute, Oberpfaffenhofen, Germany, 2008. 